\title{
Patient Medication Adherence Among Pharmacies Participating in a North Carolina Enhanced Services Network
}

\author{
Benjamin Y. Urick, PharmD, PhD; Monali Bhosle, PhD; and Joel F. Farley, PhD
}

\begin{abstract}
BACKGROUND: Improving medication adherence can reduce health care spending, and studies have demonstrated community pharmacists can positively affect adherence through the provision of enhanced services. The North Carolina (NC) Community Pharmacy Enhanced Services Network (CPESN) was formed in early 2014 with the goal of enhancing the care provided through its network pharmacies.
\end{abstract}

OBJECTIVE: To evaluate differences in medication adherence performance scores between pharmacies that participated in the NC-CPESN and control pharmacies in NC that did not.

METHODS: Medication adherence performance data for statins, reninangiotensin system antagonists, oral diabetes medications, and a custom multiple chronic medication measure were gathered from quarterly reports between December 2014 and September 2016. Data for these quarterly reports were derived from NC Medicaid claims. These data were combined with pharmacy demographics and service offerings data from the National Council on Prescription Drug Plans dataQ database. Descriptive statistics were used to evaluate differences in demographics and service offerings between study cohorts. Generalized estimating equations were used to evaluate the relationship between medication adherence and pharmacy cohorts, demographics, and service offerings.

RESULTS: There were 267 enhanced services pharmacies and 1,872 control pharmacies included in this analysis. Enhanced services pharmacies were much more likely to be independent pharmacies, located in rural counties, offer multidose compliance packaging, and offer delivery services, but were less likely to offer 24-hour emergency services. Persistently higher adherences scores were observed for enhanced services pharmacies, with differences across measures ranging from $3.0 \%$ to $7.2 \%(P<0.001)$. In multivariable models, the difference between enhanced services and control pharmacies was explained by differences in offerings of multidose compliance packaging and delivery services, which were associated with $3.4 \%-8.2 \%$ and $3.3 \%-4.0 \%$ improvements in adherence, respectively $(P<0.001)$.

CONCLUSIONS: This study found that enhanced services pharmacies had greater adherence performance scores for the NC Medicaid population. These differences appear to be due to CPESN enhanced services pharmacies' offering of multidose compliance packaging and delivery. Future work is needed to expand this analysis to other populations, as well as to explore the relationship between delivery and adherence.

J Manag Care Spec Pharm. 2020;26(6):718-22

Copyright $@ 2020$, Academy of Managed Care Pharmacy. All rights reserved.

\section{What is already known about this subject}

Medicaid enrollees have lower rates of adherence than the general population.

Community pharmacists have influence on their patients' medication adherence.

\section{What this study adds}

This study assessed correlates of pharmacy-level adherence scores for Medicaid enrollees.

Pharmacies that participated in the North Carolina enhanced services network appeared to have greater adherence scores across a range of chronic medications than those pharmacies that did not participate.

Differences in adherence were driven by service offerings (home delivery, compliance packaging), not network participation alone, and having a drive-up window was associated with lower pharmacy adherence scores

$\mathrm{M}$ edication nonadherence leads to more than $\$ 100$ billion in avoidable health care spending each year in the United States. ${ }^{1,2}$ This problem is especially concerning for Medicaid enrollees, since their sociodemographic characteristics, such as low income/poverty, low socioeconomic status, unemployment, and family disorganization, predispose them to nonadherence. ${ }^{3}$ Research has shown that pharmacy interventions, such as medication synchronization and screenings with brief interventions and referrals, can improve medication adherence and reduce health care spending. ${ }^{4-6}$ In an effort to increase pharmacists' engagement with providing enhanced services to North Carolina (NC) Medicaid recipients with chronic illness requiring medication-focused care coordination, the NC Community Pharmacy Enhanced Services Network (CPESN) was formed in early 2014. Pharmacies that participated in the network were required to agree to performance measurement, be in good standing with the board of pharmacy, agree to provide enhanced services, and use specialized care management to report patient engagement. ${ }^{7}$ The principal enhanced services delivered by pharmacists at participating pharmacies included, but were not limited to, interventions such as synchronization of a patient's chronic medication fill dates, adherence monitoring and coaching, compliance packaging, and home delivery. 
Previous work had found that NC-CPESN pharmacies that participated in an associated federally funded study had greater adherence at baseline than non-CPESN pharmacies in North Carolina. ${ }^{8}$ However, this work excluded CPESN pharmacies that did not participate in the grant-funded program. In addition, it is not known whether observed differences in adherence were associated with the effect of the network itself or were related to selection effects wherein pharmacies providing greater adherence support services were naturally attracted to the enhanced services network. Therefore, the aims of this study were to compare differences in medication adherence between all NC-CPESN pharmacies and other pharmacies in NC that did not participate in the enhanced services program (control group), as well as to explore potential determinants of performance on adherence scores.

\section{Methods}

This was a retrospective cross-sectional study that used 2 different data sources. First, medication adherence performance reports for all NC pharmacies were used as the source for pharmacy-level performance data. These reports were originally created by Community Care of North Carolina (CCNC) to support the NC-CPESN project and used NC Medicaid data to determine medication adherence for statins, oral diabetes medications (OADs), renin-angiotensin system antagonists (RASA), and a custom multiple chronic medication adherence measure. ${ }^{8}$

Adherence was measured over a rolling 12-month period using the proportion of days covered (PDC) method with the traditional cut-off of $80 \%$ PDC for dichotomizing patients as adherent or not. ${ }^{9}$ Pharmacy-level performance scores were defined as the percentage of patients eligible for the adherence measure denominator (e.g., statin users with at least 2 fills) that were adherent to medication treatment. Performance reporting began in December 2014 and continued every 3 months until the last report in September 2016, creating 8 performance periods and up to 8 different performance reports per pharmacy. Attribution for purposes of performance measurement was determined using a 3-month window preceding the performance measurement period. Additional information on performance measurement used to support this project can be found in Urick et al. (2018). ${ }^{8}$ For the current study, a pharmacy was excluded from the performance period for a given measure if it had fewer than 10 measure-eligible patients for that period.

The second source of data came from the National Council for Prescription Drug Programs (NCPDP) dataQ Pharmacy Database. ${ }^{10}$ This database contains a variety of information on the demographics and service offerings of U.S. pharmacies.

Variables for this project included the type of pharmacy (independent vs. chain) and services offered (e.g., compounding, delivery, drive-up, 24-hour services, and multidose compliance packaging). NCPDP data were linked to performance records using the pharmacy's National Provider Identifier
(NPI). NCPDP service data were available for most but not all pharmacies. An initial load of NCPDP data found that service information in the 2017 master file was only completed around $30 \%$ of the time, with more independent pharmacies reporting services than chain pharmacies. Data are entered by pharmacy staff or by corporate chain staff and, according to NCPDP, the dataQ database does not keep track of when fields are updated (D. Mammen, written communication, September 2019). A comparison with 2019 NCPDP data found that service offerings between 2017 and 2019 were highly consistent. As such, service offerings from 2017 were used as the primary source of service data. If 2017 data were not available, service offerings were imputed using 2019 data. The primary analysis only included those pharmacies that had complete data from the NCPDP records for either 2017 or 2019.

Enhanced services pharmacies were defined as those participating in the NC-CPESN program for at least 1 performance quarter (enhanced services cohort). Pharmacies could enter and exit the program at their own will, and a conservative approach was chosen wherein a pharmacy was assigned to the enhanced services pharmacy cohort if it was ever a participant in the NC-CPESN during any performance period. The control cohort was defined as all NC pharmacies that served Medicaid patients during the intervention period but never participated in the NC-CPESN.

Bivariate differences in service offerings between study cohorts were assessed using chi-square tests. Generalized estimating equations with repeated measure for pharmacy was used for all statistical models. Base statistical models with pharmacy type (enhanced services vs. control) and performance period number as independent variables and the 4 adherence outcomes as dependent variables were constructed to create unadjusted estimates of mean differences in medication adherence between enhanced services pharmacies and control cohort, controlling for time and seasonal trends. Also, fully specified models were constructed that included the independent variables from the base model, as well as demographic characteristics, service offerings from the NCPDP database, and rurality.

To create comparable estimates, pharmacies with missing data for the full model were removed from the base model. Rurality was determined using the rural-urban classification code (RUCC) for the county where the pharmacy was located and was dichotomized as rural or urban using RUCC 1-3 for urban and 4-9 for rural. ${ }^{11}$

All statistical analyses were conducted using SAS version 9.4 (SAS Institute, Cary, NC), and ethics review was conducted by the UNC Institutional Review Board.

\section{Results}

There were 267 pharmacies identified as enhanced services pharmacies, and 1,872 pharmacies that did not participate in the enhanced services network which served as controls. Data 


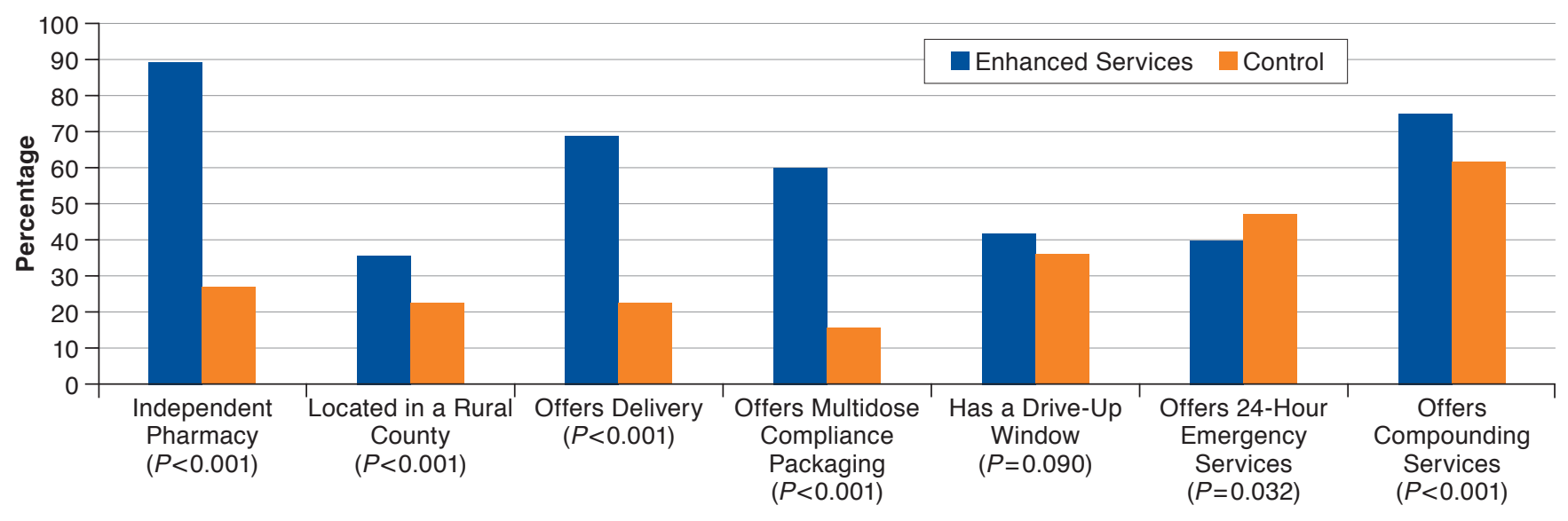

completeness varied by item in the NCPDP database. The most complete variables were status as independent or rural pharmacy, which was $100 \%$ for the enhanced services pharmacies and $99.9 \%$ for controls. The least complete variables were offering multidose compliance packaging or 24-hour emergency services, which were both approximately $88 \%$ complete for enhanced services pharmacies and 57\% complete for control pharmacies. Pharmacies with incomplete performance data were removed from analysis.

Significant differences were observed in many demographics and service offerings variables between study cohorts (Figure 1). Nearly 90\% of enhanced services pharmacies were independent pharmacies, compared with less than 30\% of control pharmacies $(P<0.001)$. Enhanced services pharmacies were also more likely than control pharmacies to be located in rural counties $(35.6 \%$ vs. $22.1 \%, P<0.001)$, offer delivery (69.2\% vs. $22.3 \%, P<0.001$ ), offer multidose compliance packaging $(59.9 \%$ vs. $16.2 \%, P<0.001)$, and offer compounding services $(74.8 \%$ vs. $61.3 \%, P<0.001)$ but were less likely to offer 24 -hour emergency services (39.4\% vs. $47.1 \%, P=0.0321$ ). No differences were observed for having a drive-up window.

After accounting for missing data and requiring at least 10 attributed patients for a performance period, the number of enhanced services and control pharmacies eligible for statistical modeling ranged from 132 and 357 for OADs to 223 and 771 for the multiple chronic medications measure (Table 1). From the base model, enhanced services pharmacies had significantly greater adherence scores on all 4 measures, with absolute differences in the percentage of attributed patients who were adherent, ranging from $3.0 \%$ for RASA to $8.2 \%$ for OADs $(P<0.001$; Table 1$)$. However, when the results from the fully specified model were assessed, the correlation between participation in the enhanced services pharmacy network and medication adherence was explained by other variables in the model. Offering delivery had a significant and positive correlation with multiple chronic medication adherence (3.3\%) and OADs (4.0\%). Multidose compliance packaging had significant and positive correlations with all adherence performance scores, ranging from $3.4 \%$ to $8.2 \%$. Interestingly, a negative correlation was observed for having a drive-up window for 3 of the 4 adherence measures (Table 1).

\section{Discussion}

This study found that enhanced services pharmacies participating in the NC-CPESN had significantly higher adherence performance scores for Medicaid enrollees than other pharmacies in NC. In addition, we found correlations between medication adherence and greater offerings of adherence support services (delivery and multidose compliance packaging), which were more common in CPESN pharmacies. These results confirm observations of greater adherence among NC-CPESN pharmacies that also participated in a related federally funded project. ${ }^{8}$ Intriguingly, higher medication adherence scores were observed for enhanced services pharmacies despite these pharmacies serving a sicker population at baseline. ${ }^{12}$

The finding that multidose compliance packaging is associated with increased adherence confirms results from studies showing that compliance packaging provided by pharmacies improves adherence. ${ }^{13,14}$ The effect of home medication delivery, however, has been much less studied. Transportation to receive health care services is a particular challenge for poor and rural patients, and that lack of transportation reduces medication filling rates. ${ }^{15}$ By offering home medication delivery, it is possible that CPESN pharmacies are overcoming transportation-related barriers, especially for the Medicaidenrolled population that comprised this study. More work is 


\section{TABLE 1 Parameter Estimates for Medication Adherence Statistical Models}

\begin{tabular}{|c|c|c|c|c|}
\hline Model Parameter ${ }^{\mathrm{a}}$ & $\begin{array}{c}\text { Multiple Chronic } \\
\text { Medication } \\
\text { Adherence }^{b}\end{array}$ & Statins & $\begin{array}{c}\text { Oral Diabetes } \\
\text { Medications }\end{array}$ & RASA \\
\hline Enhanced services pharmacy count & 223 & 192 & 132 & 200 \\
\hline Control pharmacy count & 771 & 587 & 357 & 658 \\
\hline \multicolumn{5}{|l|}{ Base model } \\
\hline Intercept & $51.8(50.7-52.8)^{\mathrm{c}}$ & $48.5(47.2-49.9)^{c}$ & $46.7(44.7-48.7)^{c}$ & $47.8(46.7-49.0) \mathrm{c}$ \\
\hline Enhanced services pharmacy (base) & $3.7 \quad(1.8-5.7)^{\mathrm{c}}$ & $4.3(2.0-6.6)^{c}$ & $8.2 \quad(5.1-11.3)^{\mathrm{c}}$ & $3.0 \quad(1.3-4.8)^{\mathrm{c}}$ \\
\hline \multicolumn{5}{|l|}{ Adjusted model } \\
\hline Intercept & $48.1(46.2-50.0)^{\mathrm{c}}$ & $45.7(43.3-48.1)^{c}$ & $44.6(41.2-48.0)^{c}$ & $46.6(44.6-48.6)$ \\
\hline Enhanced services pharmacy (adjusted) & $-2.0(-4.4-0.3)$ & $-1.4(-4.2-1.3)$ & $-0.8(-4.9-3.3)$ & $-0.2(-2.4-2.1)$ \\
\hline Independent pharmacy & $3.1 \quad(0.9-5.3)^{\mathrm{d}}$ & $2.2(-0.4-4.9)$ & $5.1 \quad(0.8-9.5)^{\mathrm{e}}$ & $1.5(-0.7-3.7)$ \\
\hline Located in a rural county & $-1.2(-2.9-0.5)$ & $0.0(-2.0-2.1)$ & $-2.1(-4.9-0.7)$ & $0.2(-1.4-1.9)$ \\
\hline Offers delivery & $3.3(1.0-5.6)^{\mathrm{d}}$ & $2.6(-0.1-5.3)$ & $4.0 \quad(0.1-7.8)^{\mathrm{e}}$ & $2.1(-0.2-4.4)$ \\
\hline Offers multidose compliance packaging & $7.2 \quad(4.7-9.6)^{\mathrm{c}}$ & $8.2 \quad(5.3-11.1)^{\mathrm{c}}$ & $7.1 \quad(3.0-11.3)^{\mathrm{c}}$ & $3.4 \quad(1.2-5.7)^{\mathrm{d}}$ \\
\hline Has a drive-up window & $-2.9(-4.5--1.2)^{\mathrm{c}}$ & $-2.8(-4.8--0.7)^{\mathrm{d}}$ & $-3.4(-6.3--0.4)^{\mathrm{e}}$ & $-1.3(-2.9-0.4)$ \\
\hline Offers 24-hour emergency services & $2.4 \quad(0.6-4.1)^{\mathrm{d}}$ & $1.6(-0.5-3.7)$ & $-1.2(-4.0-1.7)$ & $0.8(-0.9-2.5)$ \\
\hline Offers compounding services & $1.0(-0.9-2.8)$ & $0.0(-2.2-2.3)$ & $2.1 \quad(-1.2-5.4)$ & $-0.6(-2.4-1.2)$ \\
\hline \multicolumn{5}{|c|}{ 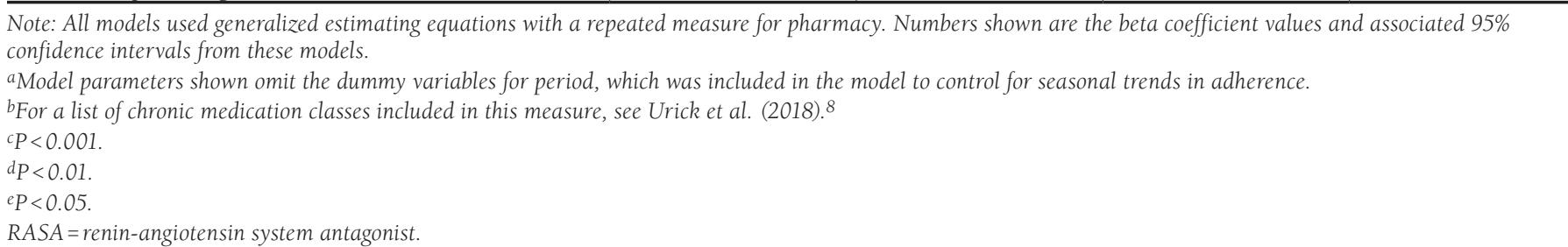 } \\
\hline
\end{tabular}

needed to explore the effect of home medication delivery on medication adherence, especially for vulnerable populations with existing transportation concerns.

The finding that independent pharmacies have greater adherence than chain pharmacies confirms a previous study's finding that patients with newly diagnosed type 2 diabetes enrolled in Medicaid had higher adherence if they filled prescriptions at an independent pharmacy. ${ }^{16}$ However, a study conducted in the Medicare population found that independent pharmacies had lower medication adherence for all of the included study measures. ${ }^{17}$ The underlying differences in Medicaid versus Medicare populations may be contributing to the conflicting results, and future research focusing on Medicare and other lines of business may shed more light on the effect of type of pharmacy on medication adherence.

The association between having a drive-up window and lower medication adherence is intriguing and has also not been extensively studied. Research has suggested that the quality of care provided through drive-up windows is lower than that provided over the counter. ${ }^{18,19}$ Given the relationship between pharmaceutical care and medication adherence, ${ }^{20}$ it is therefore possible that having a drive-up window could lower medication adherence by lessening care quality. Alternatively, it is possible that either the design or location of a pharmacy that has a drive-up window, or the preferences of patients who frequent pharmacies with drive-up windows, could contribute independently to lower measures of adherence, and this is manifesting in the model as a negative coefficient for driveup window. If additional variables on prescription volume, location, and patient characteristics were to be included in models, this could better illuminate this possibility. This type of analysis is outside the current scope of work and deserves future study.

\section{Limitations}

The primary limitation of this study is its cross-sectional nature. No causal inference can be made as to the relationship between any predictors and adherence. While evidence supports the role of multidose compliance packaging and delivery on medication adherence rates, direct causality cannot be inferred from this study. In addition, this study used pharmacy-level adherence performance scores instead of patient scores. While this aids in generalizing to performancebased payment models that seek to incentivize pharmacies for improving performance, this method does not allow for statistical adjustment for patient-level factors that may independently influence pharmacies' adherence scores.

Furthermore, NCPDP data were not available for all pharmacies in NC, and data completeness was higher for enhanced services pharmacies than for the control pharmacies. This creates 
a potential for nonresponse bias, which could not be assessed with the data available for this study. The direction of this bias depends on the selection of pharmacies that completed the survey, which cannot be determined through available data.

Another limitation is that the time frame for collection of service-related data was ambiguous. However, service offerings were highly consistent for pharmacies that could be observed in both years, and failure to properly identify service offerings would likely bias these estimates towards the null, since service offerings by pharmacies tend to increase over time. Regardless, this research generates interesting hypotheses but cannot make definitive conclusions as to the relationship between service offerings and medication adherence.

\section{Conclusions}

Our study finds that enhanced services pharmacies that participated in the NC-CPESN had higher adherence performance scores than other pharmacies in NC. This difference appears to be driven by greater offering of multidose compliance packaging and home medication delivery. Further research is needed into the causal relationship between these service offerings and medication adherence, especially the relationship between delivery and adherence. As insurers and others consider partnering with networks of pharmacies to support medication adherence, focusing on enhanced services pharmacies that offer these services may help drive program success.

\section{Authors}

BENJAMIN Y. URICK, PharmD, PhD, UNC Eshelman School of Pharmacy, Chapel Hill, North Carolina; MONALI BHOSLE, PhD, Community Care of North Carolina/CPESN USA, Raleigh; and JOEL F. FARLEY, PhD, University of Minnesota College of Pharmacy, Minneapolis.

AUTHOR CORRESPONDENCE: Benjamin Y. Urick, PharmD, PhD, UNC Eshelman School of Pharmacy, 300 Pharmacy Ln., Chapel Hill NC 27599. Tel.: 919.966.1212; E-mail: benurick@email.unc.edu.

\section{DISCLOSURES}

The project described was supported by Funding Opportunity Number 1C12013003897 from the U.S Department of Health and Human Services, Centers for Medicare \& Medicaid Services. The contents provided are solely the responsibility of the authors and do not necessarily represent the official views of HHS or any of its agencies. Ulrick reports consulting fees from Pharmacy Quality Solutions, unrelated to this work. Bhosle is an employee of Community Care of North Carolina, the not-for-profit company that sponsored the North Carolina enhanced services pharmacy network, and CPESN USA, a for-profit company that developed out of the original grant-funded project. Farley has nothing to disclose.

\section{ACKNOWLEDGMENTS}

The authors acknowledge the assistance of Troy Trygstad in the conceptualization of this project.

\section{REFERENCES}

1. IMS Institute for Healthcare Informatics. Avoidable costs in U.S healthcare: the $\$ 200$ billion opportunity from using medicines more responsibly. June 2013. Available at: http://offers.premierinc.com/ rs/381-NBB-525/images/Avoidable_Costs_in\%20_US_Healthcare-IHII_ AvoidableCosts_2013\%5B1\%5D.pdf. Accessed April 19, 2020.

2. Watanabe JH, McInnis T, Hirsch JD. Cost of prescription drug-related morbidity and mortality. Ann Pharmacother. 2018;52(9):829-37.

3. Kardas P, Lewek P, Matyjaszczyk M. Determinants of patient adherence: a review of systematic reviews. Front Pharmacol. 2013;4:91.

4. Pringle JL, Boyer A, Conklin MH, McCullough JW, Aldridge A. The Pennsylvania Project: pharmacist intervention improved medication adherence and reduced health care costs. Health Aff (Millwood). 2014;33(8):1444-52

5. Milosavljevic A, Aspden T, Harrison J. Community pharmacist-led interventions and their impact on patients' medication adherence and other health outcomes: a systematic review. Int J Pharm Pract. 2018;26(5):387-97.

6. Krumme AA, Glynn RJ, Schneeweiss S, et al. Medication synchronization programs improve adherence to cardiovascular medications and health care use. Health Aff (Millwood). 2018;37(1):125-33.

7. Smith MG, Ferreri SP, Brown P, Wines K, Shea CM, Pfeiffenberger TM. Implementing an integrated care management program in community pharmacies: a focus on medication management services. J Am Pharm Assoc (2003). 2017;57(2):217-221.el.

8. Urick B, Ferreri S, Shasky C, Pfeiffenberger TM, Trygstad T, Farley JF. Lessons learned from using global outcome measures to assess community pharmacy performance. J Manag Care Spec Pharm. 2018;24(12):1278-83. Available at: https://www.jmcp.org/doi/10.18553/jmcp.2018.24.12.1278.

9. Nau D. Proportion of days covered as a preferred method of measuring medicaiton adherence. 2012. Available at: http://ep.yimg.com/ty/cdn/epill/ pdcmpr.pdf. Accessed April 19, 2020.

10. National Council for Prescription Drug Programs. dataQ: a product of NCPDP. 2019. Available at: http://dataq.ncpdp.org/. Accessed April 19, 2020.

11. U.S. Department of Agriculture Economic Research Service. Rural-urban continuum codes. October 25, 2019. Available at: https://www.ers.usda.gov/ data-products/rural-urban-continuum-codes.aspx. Accessed April 19, 2020.

12. Urick BY, Farley JF. Do enhanced services pharmacies serve sicker patients? J Am Pharm Assoc (2003). 2019;59(2):280-84.

13. Lee JK, Grace KA, Taylor AJ. Effect of a pharmacy care program on medication adherence and persistence, blood pressure, and low-density lipoprotein cholesterol: a randomized controlled trial. JAMA. 2006;296(21):2563-71.

14. Conn VS, Ruppar TM, Chan KC, Dunbar-Jacob J, Pepper GA, De Geest S. Packaging interventions to increase medication adherence: systematic review and meta-analysis. Curr Med Res Opin. 2015;31(1):145-60.

15. Syed ST, Gerber BS, Sharp LK. Traveling towards disease: transportation barriers to health care access. J Community Health. 2013;38(5):976-93.

16. Kalsekar I, Sheehan C, Peak A. Utilization patterns and medication adherence in patients with type 2 diabetes: variations based on type of pharmacy (chain vs independent). Res Social Adm Pharm. 2007;3(4):378-91.

17. Desai V, Nau D, Conklin M, Heaton PC. Impact of environmental factors on differences in quality of medication use: an insight for the Medicare Star Rating System. J Manag Care Spec Pharm. 2016;22(7):779-86. Available at: https://www.jmcp.org/doi/10.18553/jmcp.2016.22.7.779.

18. Szeinbach S, Seoane-Vazquez E, Parekh A, Herderick M. Dispensing errors in community pharmacy: perceived influence of sociotechnical factors. Int J Qual Health Care. 2007;19(4):203-09.

19. Odukoya OK, Chui MA, Pu J. Factors influencing quality of patient interaction at community pharmacy drive-through and walk-in counselling areas. Int J Pharm Pract. 2014;22(4):246-56.

20. Jokanovic N, Tan EC, Sudhakaran S, et al. Pharmacist-led medication review in community settings: an overview of systematic reviews. Res Social Adm Pharm. 2017;13(4):661-85. 